\title{
Explosive events and transition region blinkers: Time variability of non-Gaussian quiet Sun EUV spectra
}

\author{
H. Peter and A. Brković
}

\author{
Kiepenheuer-Institut für Sonnenphysik, 79104 Freiburg, Germany
}

Received 16 December 2002 / Accepted 21 February 2003

\begin{abstract}
The transition region (TR) from the chromosphere to the corona of the Sun and solar type stars is a very dynamic regime. On the Sun at least two major observational classes of transients can be seen: explosive events and blinkers. Besides these transients there is also a more steady spectral component in transition region lines that accounts for the deviation of the line profile from a (single) Gaussian shape, i.e. for enhanced wings that are well described by a second broad Gaussian, a tail component. The present paper discusses the spectral properties of these features in order to learn more about a possible connection between blinkers, explosive events and tail components.

This paper will show that explosive events are most probably not related to the tail components or to transition region blinkers. During a blinker not only the intensity rises, as described by many CDS studies, but also the line width and shift are changing, as we show with SUMER spectra. The variation in line shift and width is closely related to the intensity variation. The line width drops to very small values at the midst of a blinker, almost reaching the thermal line width, while at the same time the Doppler shifts reach a maximum value. From this we may conclude that blinkers are driven by events in the chromosphere heating a loop asymmetrically and powering a strong laminar flow through the loop.
\end{abstract}

Key words. Sun: chromosphere - Sun: corona - Sun: transition region - Sun: UV radiation

\section{Introduction}

Our present conception of the transition region from the chromosphere to the corona is largely based on the work of Gabriel (1976) and Dowdy et al. (1986). In the former model the whole transition region is connected directly to the corona via coronal funnels, while in the latter sketch small cool and hot loops emerge between and below the funnels. In contrast to these static pictures the natural state of the transition region is dynamic. Here the shortest time-scales are observed, with very rapid changes of intensity, line shift and width down to seconds. Even in the quiet parts of the transition region short-lived not resolved small-scale events are proposed to shake the atmosphere and cause the spectral properties of the emission lines to change (e.g., 1D loop models by Hansteen 1993; Teriaca et al. 1999 or 2D models by Doyle et al. 2002). The predictions of these models for the average line shift agree well with the observations (e.g., Peter 1999). Besides these very fast variations there are also two transient transition region phenomena on time scales of minutes discussed in detail in the literature.

The first class is the long known explosive events found, e.g., by HRTS (High Resolution Telescope and Spectrograph: e.g., Brueckner \& Bartoe 1983; Dere et al. 1984). They have lifetimes of about one minute and less and often show satellite components in their spectra shifted by some $100 \mathrm{~km} \mathrm{~s}^{-1}$

Send offprint requests to: $\mathrm{H}$. Peter,

e-mail: peter@kis.uni-freiburg.de towards the blue and red. They are not necessarily related to a strong brightening. Most recent observational studies have been performed using SUMER on SOHO (Solar Ultraviolet Measurements of Emitted Radiation; Wilhelm et al. 1995), showing, e.g., that explosive events occur also in coronal holes and in active regions, being stronger in the latter ones (Pérez et al. 1999). The SUMER studies clarified that explosive events are bi-directional jets following a reconnection event, as shown by comparing observations and 2D-MHD-models (Innes et al. 1997; Innes \& Toth 1999). Sarro et al. (1999) stepped back to 1D-models, but included a treatment of non-ionisation equilibrium to calculate the resulting spectra. Recently also full 2D-models including the calculation of the spectra for non-ionisation equilibrium conditions have been performed (Roussev et al. 2001a,b; Roussev \& Galsgaard 2002).

The other major class of transition region transients is blinkers (e.g., Harrison et al. 1999; Brković et al. 2001; Bewsher et al. 2002), discovered more recently by CDS on SOHO (Coronal Diagnostic Spectrometer; Harrison et al. 1995). Blinkers are brightenings best observed in transition region lines which show an intensity increase of typically 60-80\% and last for about 5 to $30 \mathrm{~min}$. Harrison et al. (1999) argued that blinkers are due to a rise in either density or filling factor, but not due to a rise in temperature. Priest at al. (2002) presented a number of blinker scenarios, one being that blinkers might be due to a compression in the chromosphere, e.g., due to exploding granules. 
So far it is not clear if, and if yes, how blinkers and explosive events are related. Ryutova \& Tarbell (2000) compared data of SUMER, TRACE and MDI and argued that explosive events are occuring behind shock downflows due to an instability, while these shocks might be related to blinkers. Thus blinkers and explosive events would be somehow related, but driven by different physics: the intensity enhancement during a blinker being due to the shock, the explosive event due to the instability. In contrast to this conclusion Marik \& Erdélyi (2002) performed 2D numerical studies to describe the blinker in exactly the same way as an explosive event, i.e., due to reconnection. They argued that blinkers are in fact explosive events, and that they are an artifact of the poor resolution of CDS. In an observational study Winebarger et al. (2002) compared SUMER spectra of the C IV line at $1548 \AA$ showing explosive events with TRACE images in the $171 \AA$ band (containing mostly Fe IX/X lines). They found some correlation, but pointed out that it is not clear whether or not the brightening in the TRACE band was due to the coronal Fe IX/X lines or due to the brightening in the $\mathrm{O}$ VI line at $172 \AA$, which is related to the explosive event. Even if the brightening in the $171 \AA$ band would be due to the $\mathrm{Fe} I \mathrm{IX} / \mathrm{X}$ lines, the question remains open whether or not the coronal brightening is related to a blinker. Thus the fundamental question of the relation of explosive events and blinkers still remains open. It is one aim of the study in the present paper to investigate this relation from the point of view of observations.

Usually blinkers are observed with the CDS, while recently explosive events have been mostly investigated with SUMER. As SUMER has better spectral and spatial resolution than CDS we will use SUMER to investigate the spectral profiles seen during a blinker and look for signatures of explosive events during this time. Therefore, the emphasis will be on the analysis of the spectral profiles, especially on deviations from a single Gaussian profile.

Kjeldseth Moe \& Nicolas (1977) were the first to show that transition region emission line profiles are not of a single Gaussian shape, but have enhanced emission in their wings (they studied C III, C IV and Si IV). Dere \& Mason (1993) reported such profiles for $\mathrm{CIV}$ and $\mathrm{Si}$ IV, fitted the profiles with double Gaussians and discussed the contribution of the wings in terms of waves. They also excluded an opacity effect to be responsible for the line wings. Chae et al. (1998, 2000) associated line profiles with enhanced wings with blinkers and offered a hypothesis that blinkers and explosive events are due to a common underlying process. The two component character of transition region lines was recently set on a broader statistical basis by Peter (2000). He decomposed the line profile into two Gaussians, a narrow core and a broad tail component, giving a tail about a factor of three broader and a factor of three less intense than the core. The tail component is present almost everywhere in the bright network structures and its emission measure largely exceeds the one of explosive events, showing that, in general, explosive events are not responsible for the tail components. His statistical study of spatial structures of the core and tail showed that the physical origin of these components is different, and he proposed that the core component comes from small loops, while the tail component has its origin in coronal funnels, which might be either the feet of large loops or of open regions. Peter (2001) showed that the line width of the tail component increased monotonically with temperature throughout the whole transition region and that this increase is consistent with upward propagating Alfvén waves in coronal funnels.

To study the relation between blinkers and explosive events and the connection of these transient events to the tail components we will study a time series of a typical transition region line. As we need a good spectral resolution to study the shape of the line profiles we used the SUMER spectrograph. With CDS the core and tail components could not be decomposed and it is also hard to study explosive events. While blinkers are mostly observed with CDS, they are, of course, also visible with SUMER. For this study we chose the O vi (1032 $\AA$ ) line, because it can be used to investigate explosive events as well as the tail components, and because it has a very high signal-tonoise ratio on the SUMER detector. Blinkers are very prominent and well studied using Ov $(629 \AA)$ with CDS, but as the formation temperature of $\mathrm{O} v$ and $\mathrm{O} v \mathrm{vI}$ is not too different $(\log T$ of 5.38 and 5.45, respectively) the blinker in $\mathrm{O}$ VI we present in the present paper should be similar to the blinkers usually studied with CDS. Therefore, O VI $(1032 \AA)$ is an ideal choice for the present investigation of blinkers, explosive events and tail components.

\section{Observations}

SUMER has been employed to observe a quiet-Sun region at the disc centre. This paper concentrates on the analysis of O VI $1032 \AA$ formed at $\log \left(T_{\mathrm{e}} / \mathrm{K}\right)=5.45(280000 \mathrm{~K}$; peak of ion fraction). SUMER observed with detector $B$ on 25 April 1997 using the $0.3^{\prime \prime} \times 120^{\prime \prime}$ slit \#6, with a pixel size of $0.3^{\prime \prime} \times 1^{\prime \prime}$. Only part of the slit was illuminated by the solar image, resulting in a final field of view of $0.3^{\prime \prime} \times 85^{\prime \prime}$. The SUMER slit was kept at a fixed location on the solar surface by compensating for solar rotation. The width of the spectral window was $2.2 \AA$ (50 pixels). The exposure time was $15 \mathrm{~s}$ and SUMER observed for $335 \mathrm{~min}$.

We have applied all standard instrumental corrections as those for flat-field, geometric distortion, gain and dead time (see also Brković et al. 2002).

In the framework of the current study we concentrate on a small part of these data, both in space and time, that is best suited to study the multi-component spectra. We have analysed the spectra taken in a bright network region of the quiet Sun (the SUMER spatial pixel \# 79) and concentrate on about 35 min (starting from 13:57 UT). These spectra are shown in Fig. 1. As it will turn out later, at this spatial location a blinker can be seen. Almost all the spectral profiles in this region in space and time fulfill the criteria used by Peter $(2000,2001)$ that a core, as well as a tail component, is present (see Sect. 4, cf. Fig. 2).

\section{Multi-component spectra: Steady or transient}

When investigating the nature of the tail components of transition region lines one is confronted with a severe signal-to-noise 


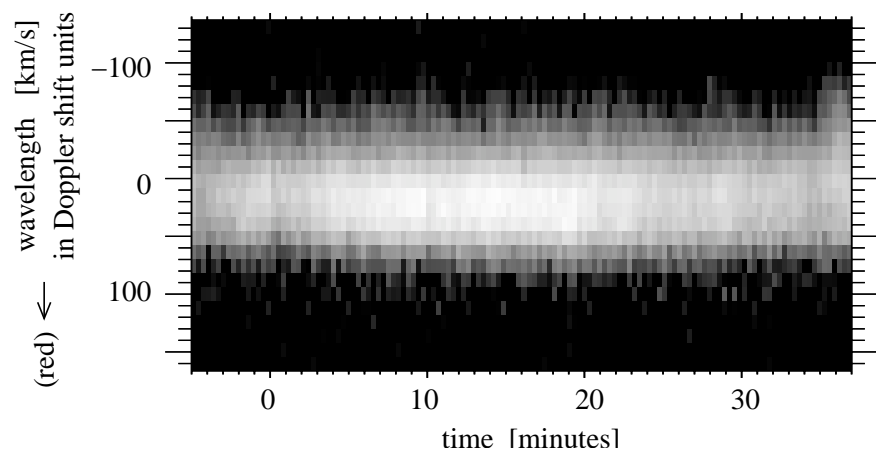

Fig. 1. Part of the time series in O vI (1032 $\AA$ ) under study in this paper for one given spatial position along the slit. The wavelength is given in units of Doppler shift. The time evolution is relatively smooth. These spectra show a double Gaussian line profile, an example being shown in Fig. 2. The spectrum is shown on a logarithmic scale.

problem. As reported by Peter (2001) a signal-to-noise or lineto-continuum ratio of at least 30 is needed to detect a broad spectral component below a three times narrower and three times brighter core component. These are typical values for the tail components as found in quiet Sun network structures (Peter 2000, 2001). For typical lines as observable with current instrumentation (here: SUMER) this implies exposure times of $90 \mathrm{~s}$ or longer.

Already Dere \& Mason (1993) and also more recently Chae et al. (1998) interpreted the tail components as being due to "classical" explosive events as discussed e.g., by Dere et al. (1989), which have lifetimes of up to $1 \mathrm{~min}$. When being observed with an exposure time of $150 \mathrm{~s}$, as done by Peter (2000), a small short explosive event might appear as a broad component in the spectrum. Peter (2000) argued that the rate of occurrence and the emission measure observed in the "classical" explosive events is not sufficient to explain the tail components, which are present almost everywhere in the network. But, of course, smaller explosive events, if occurring at a much higher rate, might well cause the observed tail components.

\subsection{Single vs. double Gaussian fits at short exposure times}

To investigate this possibility we carried out the following experiment. We binned the data in a running mean fashion to obtain spectra with effective exposure times of $15,30,45, \ldots, 150 \mathrm{~s}$ at any given time for each spatial location and then applied a single and a double Gaussian fit to each of these spectra. We have used a Genetic Algorithm based optimisation method of Charbonneau (1995). This can be considered as studying the spectra with different "effective" exposure times, $\tau_{\text {eff }}$. Some of these binned spectra are shown along with the fits in Fig. 2. They sort of represent the spectrum with various degrees of smoothing in time. This binning enhances the count rate and by this the signal-to-noise ratio, as is clearly evident from Fig. 2. (Nevertheless we show the intensity in counts per pixel and second so that the spectra are comparable.)
The fits can be checked quantitatively by using

$$
\chi_{\mathrm{r}}^{2}=\frac{1}{N-f} \sum_{i=1}^{N} \frac{\left(d_{i}-m_{i}\right)^{2}}{\sigma_{i}^{2}}
$$

where $d_{i}$ and $\sigma_{i}$ are the data and their error estimate (here Poisson errors, $\sigma_{i}^{2}=d_{i}$ ) for $N$ data points and $m_{i}$ are values for the model with $f$ degrees of freedom (here 4 or 7 for a single and a double Gaussian fit with continuum).

For the $150 \mathrm{~s}$ exposure time (Fig. 2c) it is clearly evident that a double Gaussian fit is superiour to a single Gaussian fit, both from comparing fits and data by eye and by comparing the values of the reduced $\chi_{\mathrm{r}}^{2}$ of 1.1 and 2.3 (Fig. 2d). The values of $\chi_{\mathrm{r}}^{2}$ for the single Gaussian fits decrease as the exposure time $\tau_{\text {eff }}$ decreases. This effect is simply due to the fact that the count rates are lower, implying that the relative (Poisson) errors increase. This is a general property of $\chi_{\mathrm{r}}^{2}$, as can be seen from the above definition.

If the model is a good representation of the data and the error estimates $\sigma_{i}$ are correct, $\chi_{\mathrm{r}}^{2}$ should be near unity. The model is not correct, if for good error estimates $\sigma_{i}$ the value of $\chi_{\mathrm{r}}^{2}$ largely exceeds 1 . A value of $\chi_{\mathrm{r}}^{2}$ much lower than 1 indicates that the errors $\sigma_{i}$ are too large for a reliable fit of the data with the model.

This shows that the smaller values for $\chi_{\mathrm{r}}^{2}$ for shorter exposure times $\tau_{\text {eff }}$ do not imply that the single Gaussian fits improve for smaller $\tau_{\text {eff }}$. It just reflects the fact that very short exposure times $\left(\tau_{\text {eff }}<30 \mathrm{~s}\right)$ do not allow for a really reliable fit of this line. The $\chi_{\mathrm{r}}^{2}$ for the double Gaussian fits, however, are (almost) constant for exposure times down to $45 \mathrm{~s}$ and only then drop below unity (Fig. 2d).

Therefore, one can conclude that based on the values of $\chi_{\mathrm{r}}^{2}$ a double Gaussian fit is a good fit to the data and is certainly superiour to a single Gaussian fit down to time scales of $40 \mathrm{~s}$. For shorter time scales the present instrumentation does not allow us to decide between the single and the double Gaussian fit, even though at $\tau_{\text {eff }}=15 \mathrm{~s}$ the $\chi_{\mathrm{r}}^{2}$ are 0.55 and 0.35 , thus probably favouring the double Gaussian. This is supported by a (subjective) comparison by eye of the single and the double Gaussian for the shortest exposure time (15 s, Fig. 2a). These results hold not only for the sample spectra shown in Fig. 2, but this trend can be seen with (almost) all the spectra studied here.

This discussion shows that if processes of the explosiveevent-type, or any other transient events, should explain the tail components as commonly observed in the network, they must have lifetimes on the time scale of $10 \mathrm{~s}$ or less. If they would last longer they should show up in the data analysed here as individual narrow components and not as one broad weak component. On the nature of these hypothetical short lived events one could only speculate by now.

\subsection{Relation to "classical" explosive events}

To emphasise that a "classical" explosive event is not responsible for the tail component of transition region spectra we will compare spectra with and without explosive events, that have sufficient time resolution to resolve the explosive events. 

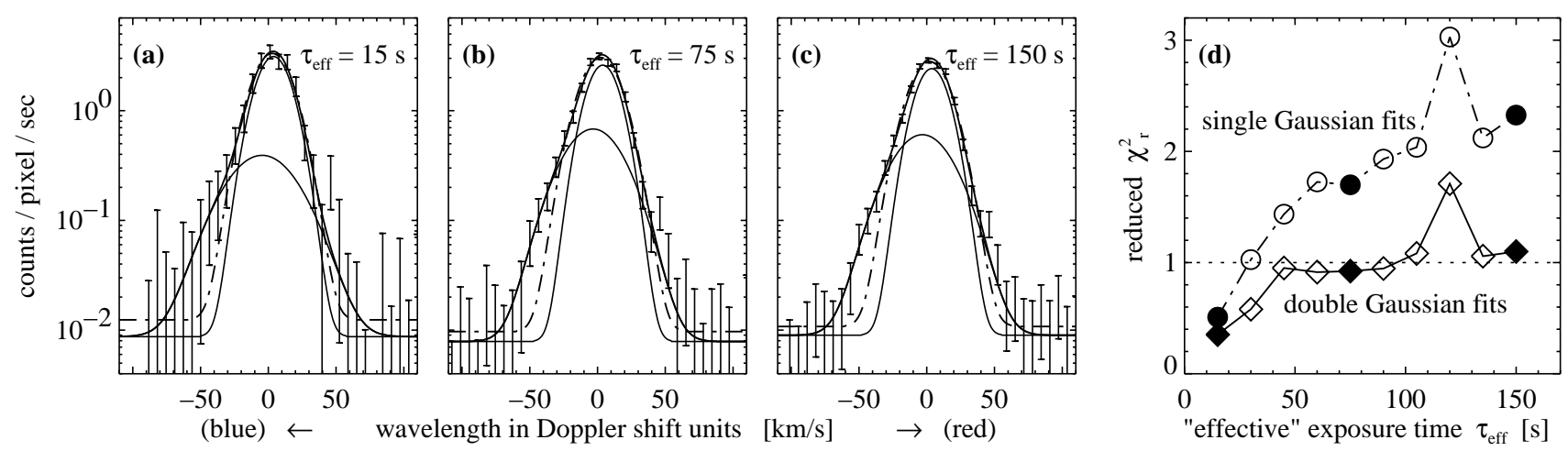

Fig. 2. The three left panels a)-c) show a spectrum of $\mathrm{O}$ vi (1032 ̊̊) taken from the time series shown in Fig. 1 at the time 31 min for different "effective" exposure times $\tau_{\text {eff }}$ (i.e., temporal binning in a running mean fashion with different box sizes $\tau_{\text {eff }}$; the values for $\tau_{\text {eff }}$ are given with the plots). The observations are shown as bars that represent the (Poisson) errors of the count rate. The single Gaussian fits are shown as dotted-dashed lines, the double Gaussian fits as thick solid lines, with the two components of the double Gaussian being plotted thin solid. The right panel d) shows the values of $\chi_{\mathrm{r}}^{2}$ for the single (circles) and double Gaussian fits (diamonds) for different binning, i.e. for different $\tau_{\text {eff }}$ (symbols for those $\tau_{\text {eff }}$ shown in the left panels are filled). See Sect. 3.1.

Figure 1 displays the data we discussed before showing the tail components in $\mathrm{O}$ vi at $1032 \AA$, while Fig. 4 shows a time series containing a "classical" explosive event in the same line (SUMER data from 28 March 1996; the time and wavelength scales are exactly the same in both figures). In the explosive event spectra one can even distinguish the two satellites to the blue and red. It is broadly accepted that these are due to a bidirectional flow caused by reconnection (e.g. Innes et al. 1997). This clearly shows the dramatic observational difference between explosive events and the tail components, when observed with sufficient spectral resolution.

To further investigate the relation of the tail components to explosive events we also applied a triple Gaussian fit to the spectra shown in Fig. 1 that have been analysed in terms of double Gaussians so far. We constrained the triple Gaussian fits by the physical picture we have of explosive events. If an explosive event is a bi-directional jet following a reconnection event one expects three spectral components: a line core representing the background, and two satellites shifted towards the red and blue due to the flow in the event. As the direction of the flow is arbitrary one should expect the satellites to show any offset from $0 \mathrm{~km} \mathrm{~s}^{-1}$ to the Alfvén speed (about 100 to $500 \mathrm{~km} \mathrm{~s}^{-1}$ in the transition region). These satellites have, however, a line width comparable to the normal quiet Sun line core (cf. e.g. 1D model by Teriaca et al. 1999; 2D-MHD calculations of Roussev et al. 2001a,b and Roussev \& Galsgaard 2002). The plasma we see in the spectral satellites, i.e. surrounding the very small reconnection site, is accelerated by the reconnection, while the heating is concentrated in the reconnection region itself.

Based on this physical argument we chose the constraints for the triple Gaussian fits as follows: there is one core component near the rest wavelength and two further components. The line widths of all three components have to be close to the line width of the core at normal quiet Sun network, i.e. $34 \pm$ $3 \mathrm{~km} \mathrm{~s}^{-1}$ (Peter 2001). Thus each component is allowed to have a line width from 31 to $37 \mathrm{~km} \mathrm{~s}^{-1}$. The intensity of the satellites and their position is not constrained, except that one of the satellites has to be blueward, the other one towards the red of the core, but the shift must not necessarily be symmetric. In Fig. 3a, a double Gaussian fit for a sample spectrum is shown $\left(\tau_{\text {eff }}=150 \mathrm{~s}\right)$ and Fig. $3 \mathrm{~b}$ shows a triple Gaussian fit for the same profile.

The comparison of the results for the double and the (constrained) triple Gaussian fits shows that the $\chi_{\mathrm{r}}^{2}$ for the double Gaussians is systematically lower than the one for the triple Gaussians. Figure 3c shows the distribution of $\chi_{\mathrm{r}}^{2}$ for the 100 spectra (corresponding to $35 \mathrm{~min}$ ) of the time series shown in Fig. 1. This shows that the double Gaussian fits are a good representation of the data as they follow the theoretical optimal curve (e.g. Bevington \& Robinson 1992). The constrained triple Gaussian fits, mimicking an explosive event situation, however, are worse than the double Gaussians.

This discussion shows that the tail components of transition region lines and the classical explosive events are most probably not related to each other. However, it is possible that the tail components are due to a multitude of not resolved small events, much smaller and with much shorter lifetimes than the explosive events. In contrast to the explosive events, the interpretation of Peter $(2000,2001)$ that the tail components are due to waves from coronal funnels is consistent with the present data.

\section{Time series of multi-component spectra: Transition region blinkers}

Figure 1 shows the time series under study for pixel \# 79 along the SUMER slit. As the neighbouring three pixels of that network element show roughly the same temporal evolution we do not display them here (i.e. four SUMER pixels corresponding to $4^{\prime \prime}$ show the time variability discussed in the following). Almost all the spectra (95\%) out of these 35 min show a double Gaussian line profile with a narrow core and a broad tail component when applying the same criteria as in Peter (2000, 2001): A double Gaussian profile is only then considered as being significant (i.e. better than a single Gaussian) if (1) $\chi_{\mathrm{r}}^{2}$ of the double Gaussian is less than 0.9 times the value for the single Gaussian fit, and (2) $\chi_{\mathrm{r}}^{2}$ for the fit is below 1.5, 

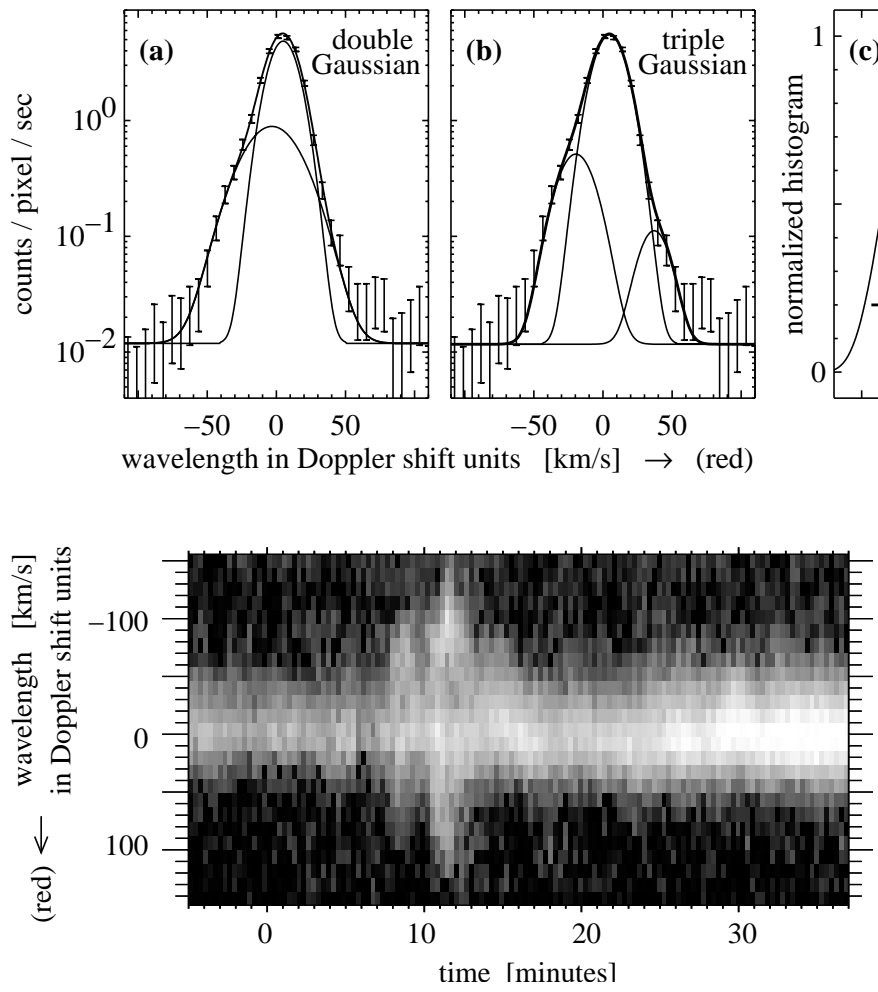

Fig. 4. Time series in $\mathrm{O}$ vi $(1032 \AA$ ) showing two explosive events (at 8 and $11 \mathrm{~min}$ ). For a better comparison the scaling in time and wavelength is exactly the same as in Fig. 1. The wavelength is given in units of Doppler shift. The spectrum is shown on a logarithmic scale. (Sect. 3.2).

i.e. the fit has to be reliable (cf. the histogram of $\chi_{\mathrm{r}}^{2}$ for the double Gaussian fits shown in Fig. 3).

We applied a double Gaussian fit to every spectral profile during the time series. To ensure a sufficient signal-to-noise ratio we binned the data in time in a running mean fashion resulting in an effective exposure time of $150 \mathrm{~s}$ (cf. Sect. 3.1), i.e., the results discussed below are based on data as the sample spectrum shown in Fig. 2c. The results do not change significantly for less binning.

The line parameters, i.e. total intensity, line width and line shift for the core (thick) and the tail component (thin) for the profiles of the time series shown in Fig. 1 are displayed in Fig. 5. Panel a also shows the total intensity of the line, i.e. core plus tail, as a dotted-dashed line. In Panel $b$ the line width corrected for instrumental broadening, $w_{1 / e}$, is shown, i.e. the Gaussian half-width at $1 / e$ of maximum value. Panel $\mathrm{c}$ shows the Doppler shifts.

The light curve is typical for a blinker (Fig. 5a) and will be discussed in Sect. 4.2.

We corrected for the instrumental broadening using the SUMER standard IDL procedure con_width funct 2 giving the line width $w_{1 / e}$. The non-thermal broadening is then given by $w_{\mathrm{nt}}^{2}=w_{1 / e}^{2}-w_{\mathrm{th}}^{2}$, with the thermal width given through $w_{\mathrm{th}}^{2}=2 k_{\mathrm{B}} T / m$, with the line formation temperature $T$ and the ion mass $m$. It should be noted that the non-thermal width depends, among other factors, on the spatial resolution. Therefore we prefer to plot the line width $w_{1 / e}$.
The evolution of the line width during the blinker should be compared to mean quiet Sun values, which will be recalled briefly. For the $\mathrm{O}$ vi line core component the line width $w_{1 / e}$ (corrected for instrumental broadening) is about $34 \mathrm{~km} \mathrm{~s}^{-1}$ in the quiet Sun network (Chae et al. 1998; Peter 2001). This value is shown by a horizontal dashed line in Fig. 5b. This corresponds to a full width at half maximum of $F W H M=$ $2 \sqrt{\ln 2} w_{1 / e} \approx 56 \mathrm{~km} \mathrm{~s}^{-1}$. The non-thermal width of $\mathrm{O} \mathrm{VI}$ at the quiet Sun network is about $29 \mathrm{~km} \mathrm{~s}^{-1}$ as the thermal width of $\mathrm{O}$ vI is $w_{\text {th }} \approx 17 \mathrm{~km} \mathrm{~s}^{-1}$ (this thermal width is shown by a horizontal dotted line in Fig. 5b).

We have calibrated the wavelength in the following way. The time immediately before the blinker reflects just regular quiet Sun (network) conditions. Therefore we calibrated the wavelength so that the average line shift during these approx. 30 min equals the mean quiet Sun Doppler shifts as reported in previous studies (about $6 \mathrm{~km} \mathrm{~s}^{-1}$ towards the red; Brekke et al. 1997; Chae et al. 1998; Peter \& Judge 1999; Teriaca et al. 1999). The mean quiet Sun disc centre redshift for O VI is shown in Fig. 5c as a horizontal dashed line. This procedure is sufficient, because we are not interested in the exact determination of the absolute Doppler shifts, but in the change during the blinker.

\subsection{Temporal evolution of multi-component spectra}

The tail component (thin lines in Fig. 5) shows little variation with time - the variations are well within the error bars. In contrast the line core (thick lines) shows a significant variation in all three line parameters. The correlation coefficients between the respective line parameters for the core and tail component are given with the plots and are all small (all have absolute values below 0.5). This agrees well with the result of Peter (2000) who found no spatial correlation between the two components. This strengthens the argument that the tail and the core component are of different physical origin.

\subsection{A blinker in detail}

The line intensity of the core component (Fig. 5a, thick line) shows a variation as it is typical for a transition region blinker. The intensity rises by a factor of about 4 and the brightening lasts for a bit less than $30 \mathrm{~min}$, thus it would be automatically detected as a blinker when using the more or less standard 

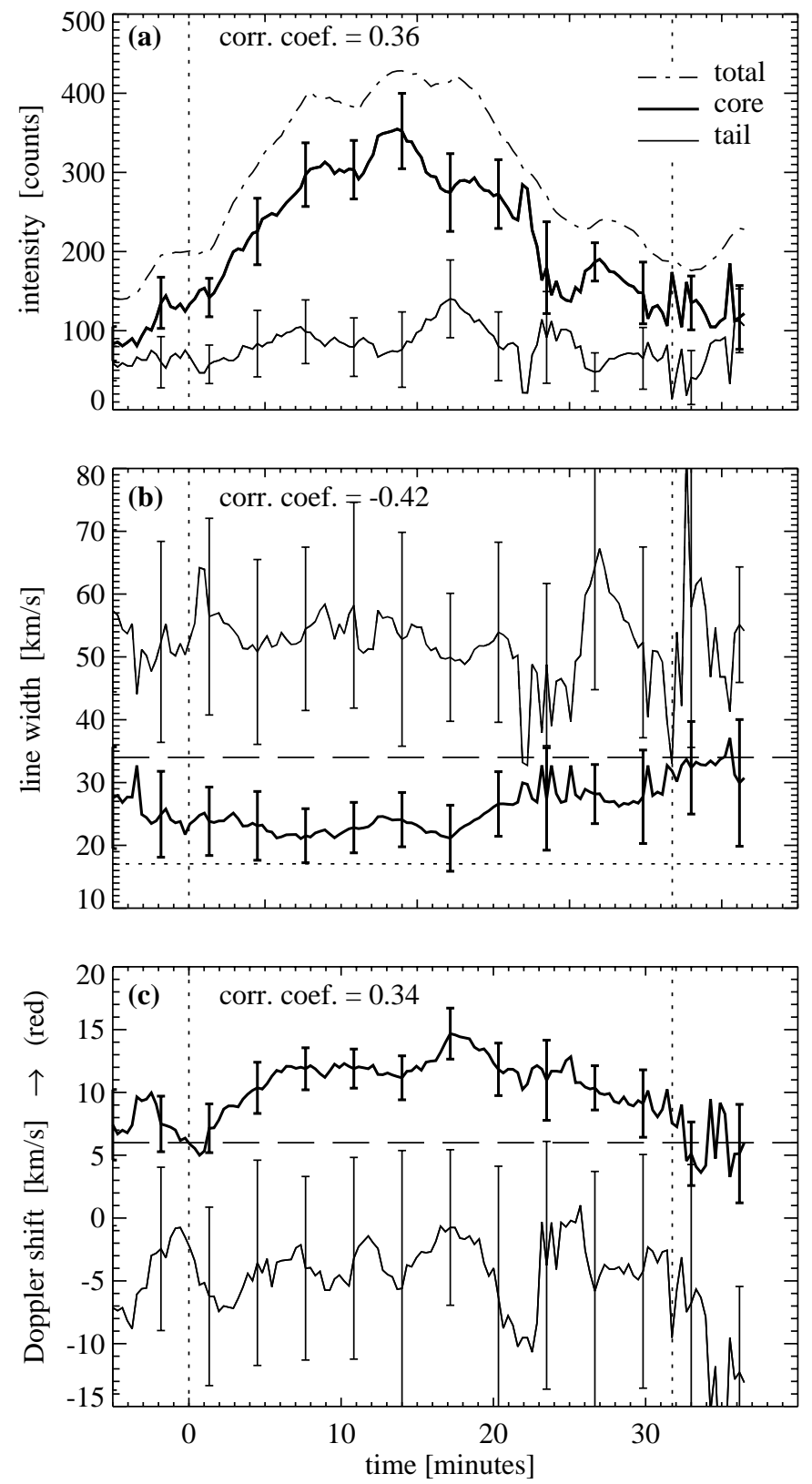

Fig. 5. Line parameters of the double Gaussian fits at a location in the network $\left(0.3^{\prime \prime} \times 1^{\prime \prime}\right.$; same location as in Fig. 1$)$. The variations for the line core component are shown as thick lines, for the tail component as thin lines. The dotted-dashed line in panel a) shows the total intensity, i.e. core plus tail. The horizontal dashed lines in panel b) and c) show typical quiet Sun at disc centre values of line width and shift for the core component. The horizontal dotted line in panel $\mathbf{b}$ ) indicates the thermal velocity of $17 \mathrm{~km} \mathrm{~s}^{-1}$ for $\mathrm{O}$ vI. The correlation coefficients between the core and tail parameters are given with the plots; there is no significant correlation between line core and tail. The errors (bars) are shown only every 3 min to avoid confusion. The vertical dotted lines indicate the begin and end of a transition region blinker. See Sect. 4.

procedures applied to CDS intensity data. It is important to note that this enhancement is seen in four subsequent SUMER pixels along the slit, which corresponds to a spatial extent of 4 ". Unfortunately, there is no information of the blinker in the direction perpendicular to the slit.
In Fig. 5 we show the variation only during the blinking event and for another $5 \mathrm{~min}$ before and afterwards, as the parameters show only small variations before and after. In contrast to CDS data the SUMER data presented here allow us also to study the changes in the line profile during the blinker event in detail.

As seen from Fig. $5 \mathrm{~b}$ the line width of the core component is dropping well below the normal quiet Sun value right at the onset of the blinker and reaches almost the thermal width: the minimum of the line width is only about $20 \mathrm{~km} \mathrm{~s}^{-1}$ compared to the thermal speed of $17 \mathrm{~km} \mathrm{~s}^{-1}$. Considering the uncertainties of the fit, it is possible that the line width is completely given by the thermal width (at times when the error bars drop below the horizontal dotted line indicating the thermal width). During the mid of the blinker event there are (almost) no unresolved nonthermal motions! Towards the end of the blinker the line width increases again, finally reaching the typical quiet Sun value.

The Doppler shift shows the opposite variation (Fig. 5c). While being typically shifted $6 \mathrm{~km} \mathrm{~s}^{-1}$ to the red, the redshift increases during the blinker reaching $15 \mathrm{~km} \mathrm{~s}^{-1}$ in the midst of the blinker. It should be noted that this is only the lineof-sight component; the actual flow velocity might be much higher, depending on the angle we are looking at the structure. Afterwards the redshift decreases again to its normal value. It is remarkable that the redshift starts decreasing at almost exactly the same time when the line width is increasing again.

During the blinker the general trends in line shift and width are closely related to the intensity variation. This is reflected by the high correlation coefficients during the rising phase of the blinker between intensity $I$ and line shift $v$ as well as line width $w(I \sim v:+0.93, I \sim w:-0.81)$. When considering the whole duration of the blinkers the values are somewhat lower $(I \sim v$ : $+0.71, I \sim w:-0.61)$.

These observational results will be discussed in Sect. 5.3.

\section{Discussion}

\subsection{Blinkers and explosive events}

As discussed already in Sect. 3, during the time of the blinker shown in Figs. 1 and 5 no explosive events have been seen. If they would be present, the spatial and temporal resolution would have allowed us to detect them. However, it might still be possible that very small transient events of lifetimes below $30 \mathrm{~s}$ are causing the tail components of the transition region spectra. (Even though we favour the interpretation of Peter 2001 that the tail components are due to coronal funnels at the feet of large coronal loops.)

Marik \& Erdélyi (2002) suggested from their models that blinkers and explosive events are essentially the same. It has to be noted, however, that their model calculation covered only $6 \mathrm{~min}$, which is on the extreme low end of the distribution of blinker durations. They argued that the CDS-blinkers are just an artifact of the poor resolution of CDS. However, using the SUMER spectrograph, the spectral evolution of the blinker under study here (Fig. 1) is radically different from an explosive event (Fig. 4). To check how the spectra would look like when observed with CDS, we degraded the spectral resolution of the 

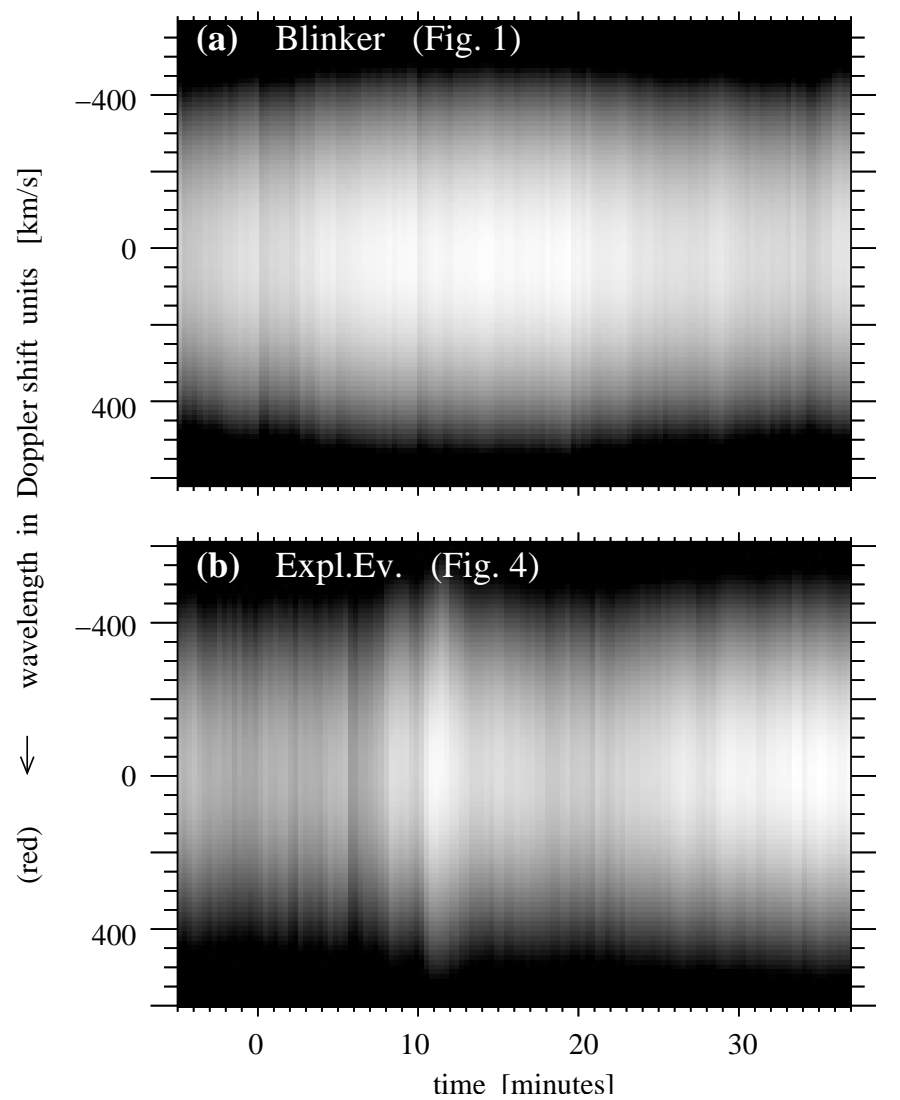

Fig. 6. Time series from Fig. 1 (blinker, top) and Fig. 4 (explosive event, bottom) obtained with SUMER, but degraded in spectral resolution to match the spectral resolution of CDS. This was done by convolving the SUMER spectra with a Gaussian of the width of the CDS instrumental broadening. The degraded spectra look much alike, i.e., CDS would not be able to distinguish between a blinker and a large explosive event. Please note the compressd wavelength scale compared to Figs. 1 and 4. (See Sect. 5.1).

spectra in Figs. 1 and 4 down to the CDS resolution. This was done by convolving them with a Gaussian with a width corresponding to the instrumental broadening of CDS. As the CDS line width is dominated by the instrumental broadening (e.g. Harrison et al. 2002) we used a minimum value of the observed line width as the instrumental width, i.e., $400 \mathrm{~m} \AA$ FWHM corresponding to $190 \mathrm{~km} \mathrm{~s}^{-1}$ for the typical CDS-blinker line $\mathrm{O} \mathrm{v}$ at $629 \AA$. The degraded spectra of the blinker and the explosive event look very much alike (Fig. 6), i.e., indeed CDS cannot distinguish between a strong explosive event and a blinker. Therefore maybe a lot of CDS-blinkers, especially those with shorter lifetimes, might be explosive events, but there is definitely a class of blinkers that does not share the spectral characteristics of explosive events, i.e., enhancements of intensity without additional spectral satellites.

At first sight this class of blinkers might nevertheless be similar to explosive events, with the bi-directional flow of the explosive event being perpendicular to the line-of-sight. Then we should see only a brightening without the additional spectral satellites. However, this idea has two major flaws: why do we see an increase in redshift, but no blue component, and especially: why is the line width decreasing during the blinker?
During a reconnection event like a large explosive event we should expect not only a channeling of the plasma into a bidirectional flow, but also a lot of not resolved small scale motions related to the reconnection site. Thus we should expect an increase of non-thermal line width during the event, in contrast to the observations. Therefore it does not seem to be likely that the blinker discussed here is just a large explosive event.

\subsection{Blinkers and tail components}

When interpreting the tail components as being due to small explosive events, these cannot account for the blinking activity, though, because the tail component shows only a very small variation in time (cf. Fig. 5a). If the tail components would be due to explosive events and if these are causing the blinker, one should see a considerable variation of the tail component, which is not seen.

In contrast, almost all variability in intensity is due to the changes in the line core component - at least this blinker is a line core phenomenon. And, as the line width of the core component is collapsing to almost the thermal width, there is little room to interpret the blinkers as being due to small scale transients, such as explosive events or other small scale reconnection events that would lead to severe not-resolved motions and by this to a strong non-thermal line broadening.

\subsection{The nature of blinkers}

Based on the results outlined in Sect. 4.2 we propose the following scenario. The region of the atmosphere that hosts the blinker is a cool coronal loop that is heated asymmetrically. This heating then leads to an increasing intensity and will drive a flow through the loop. Such a flow can lead to an increasingly strong redshift as has been shown in early loop models e.g., by McClymont (1989). The loop has to be a cool loop (well below $10^{6} \mathrm{~K}$ ) because hardly any blinker-type events have been reported at coronal temperatures.

The fact that the line width is dropping almost to the thermal width is a very hard constraint for the explanation of blinkers. It tells us that we must not expect the driver of the blinker to be located in the transition region (or at least, in the present case, not at the temperatures where $\mathrm{O}$ VI is formed). If it would, the heating should lead to some non-thermal broadening of the line as any heating mechanism would naturally lead to not resolved small scale motions. Therefore, the driver for the blinker should be located either above or below the transition region. As no signatures of blinkers are found in the corona, it seems natural to place the driver for the blinkers in the chromosphere (or below). This agrees well with (at least) one scenario that Priest et al. (2002) derived from discussing various theoretical arguments. Furthermore this is supported by recent coronal loop studies providing evidence that the heating is concentrated at the footpoints (see the recent observations from Aschwanden 2001 as well as the recent model by Spadaro et al. 2003 and references therein).

The very small line width, very close to the thermal width, confronts us with another fundamental question: how does the 
Sun avoid a non-thermal broadening over an area larger than $0.3^{\prime \prime} \times 1^{\prime \prime}$ (i.e. one spatial pixel of SUMER) for $10 \mathrm{~min}$ or more? Under regular quiet Sun conditions almost every location in time and space shows non-thermal broadening, no matter if it is in the photosphere, chromosphere or transition region. These numbers are especially impressive when comparing them to the mean cell size of the granulation of about $1.75^{\prime \prime}$ and to the lifetime of granules of 6 min (e.g., Stix 2002). Thus during a blinker the non-thermal motions are suppressed on spatial and temporal scales of the granulation! (This is not to propose a physical connection, it is just for comparison.)

One might think of a situation when the asymmetric heating of the loop causing the blinker drives a very regular laminar flow through the loop. As the Doppler shift during the blinker reaches considerable values this might be indeed the case and as long as the flow is laminar the line width remains very small. This would also imply that the blinker is a quite large scale phenomenon with a spatial structuring of not much smaller that 1 ".

In the present case the blinker has a spatial extent along the SUMER slit of about 4" (cf. beginning of Sect. 4). As we have no context image in the transition region for the instant of the blinker we will simply assume that the blinker is a brightening of a semi-circular loop which has a footpoint distance of about 4 " corresponding to $3 \mathrm{Mm}$ on the Sun. This implies a loop length of less than $10 \mathrm{Mm}$. As the Doppler shift associated with the blinker increases by about $10 \mathrm{~km} \mathrm{~s}^{-1}$ during the blinker (Fig. 5, thick line), we will further assume that the flow through the loop has a velocity of at least $10 \mathrm{~km} \mathrm{~s}^{-1}$. Probably the flow speed is higher, as the Doppler shift gives only the line-of-sight component.

The results of the present paper suggest that the driver of the blinker is to be found in the chromosphere, and Harrison et al. (1999) argued that a rise in density might cause the increased intensity of the blinker without a change of temperature. A combination of these findings leads to a scenario, where enhanced density in the chromosphere is used to fill a loop that remains at more or less the same temperature. For the above values of $10 \mathrm{Mm}$ loop length and a flow through a loop of $10 \mathrm{~km} \mathrm{~s}^{-1}$ this gives a typical time to fill the loop of $1000 \mathrm{~s}$, or $15 \mathrm{~min}$. Thus one would expect the loop to brighten up within about $15 \mathrm{~min}$. This very simple estimation for the $4^{\prime \prime}$ blinker analysed here corresponds very well to the rise time of the intensity (cf. Fig. 5).

Further combined studies of the connection of blinkers to the chromosphere are needed both theoretically and observationally.

\section{Conclusions}

We presented a time series of transition region spectra from the O VI (1032 $\AA$ ) line, whose profiles consist of a narrow core and a weak and broad tail component. The broad tail component was detected for exposure times as short as $45 \mathrm{~s}$. Double Gaussian fits (i.e. core and tail) to the spectral profiles are also superiour to triple Gaussian fits for an explosive event type situation. From this we conclude that the wings of transition region lines are not due to explosive events as proposed by Dere \& Mason (1993) or Chae et al. (1998).
This study showed that with CDS, which is usually employed to study blinkers, one cannot distinguish between a strong explosive event and an intensity rise without spectral satellites. Thus some of the transition region brightenings classified by CDS studies as blinkers, might be in fact large explosive events. Though, this would apply only to very short-living CDS-blinkers. Using SUMER data with their higher spectral resolution we showed that strong increases in intensity over the typical time scale of a blinker happen with significantly different spectral properties than those of explosive events.

During a blinker we see a strong increase of the line core intensity, but hardly any signature in the line parameters for the tail component (within the errors) - blinkers are line core events. Therefore, even if the line tail would be due to explosive events, there would be no correlation between explosive events and blinkers.

During the course of the blinker not only the line core intensity rises, but also the core line width is dropping to almost thermal widths and the redshift is increasing to substantial values. Especially the very small line width, being close to the thermal width puts a very tight constraint on explanations of transition region blinkers.

We propose that a blinker is caused by a steady asymmetric heating of a cool coronal loop at the chromospheric base of the loop. This leads to a strong laminar flow through the loop with no substantial non-thermal broadening. The observation of the almost thermal line width might be a hint that we are about to resolve a blinker, i.e., it has a spatial scale of about $1^{\prime \prime}$ or more.

No matter whether or not our interpretation of the small line widths will hold, we think that the decrease of the core line width to its pure thermal counterpart is of pivotal importance for future modeling of explosive events. Except for some lines observed in the photosphere of sunspots this is one of the very rare cases on the Sun where one observes a spectral line at its thermal width.

Further studies on the variation of the line profile parameters during a blinker, especially at different temperatures, are needed to draw further conclusions. This study shows that we will need more spectroscopic investigations with sufficient spectral resolution to really understand transition region blinkers.

Acknowledgements. We would like to thank Rolf Schlichenmaier for fruitful discussions and we are grateful to the SUMER team and its open data policy. Sincere thanks are due to the anonymous referee for helpful comments. The SUMER project is financially supported by DLR, CNES, NASA and the ESA PRODEX programme (Swiss contribution). SOHO is a mission of international cooperation between ESA and NASA. AB thanks J. O. Stenflo and S. K. Solanki for encouragement and support. The work of $\mathrm{AB}$ was supported by the Deutsche Forschungsgemeinschaft, which is gratefully acknowledged.

\section{References}

Aschwanden, M. J. 2001, ApJ, 560, 1035

Bevington, P. R., \& Robinson, D. K. 1992, Data Reduction and Error Analysis for the Physical Sciences (New York: McGraw-Hill)

Bewsher, D., Parnell, C. E., \& Harrison, R. A. 2002, Sol. Phys., 206, 
Brekke, P., Hassler, D. M., \& Wilhelm, K. 1997, Sol. Phys., 175, 349 Brković, A., Solanki, S. K., \& Rüedi, I. 2001, A\&A, 373, 1056 Brković, A., Solanki, S. K., \& Rüedi, I. 2002, A\&A, 385, 257 Brueckner, G. E., \& Bartoe, J.-D. F. 1983, ApJ, 272, 329 Chae, J., Yun, H. S., \& Poland, A. I. 1998, ApJS, 114, 151 Chae, J., Schühle, U., \& Lemaire, P. 1998, ApJ, 505, 957 Chae, J., Wang, H., Goode, P. R., et al. 2000, ApJ, 528, L119 Charbonneau, P. 1995, ApJS, 101, 309

Dere, K. P., \& Mason, H. E. 1993, Sol. Phys., 144, 217

Dere, K. P., Bartoe, J.-D. F., \& Brueckner, G. E. 1984, ApJ, 281, 870 Dere, K. P., Bartoe, J.-D. F., \& Brueckner, G. E. 1989, Sol. Phys., 123, 41

Dowdy, J. F. Jr., Rabin, D., \& Moore, R. L. 1986, Sol. Phys., 105, 35

Doyle, J. G., Madjarska, M. S., Roussev, I., et al. 2002, A\&A, 396, 255

Gabriel, A. H. 1976, Phil. Trans. Roy. Soc. London A, 281, 339

Hansteen, V. H. 1993, ApJ, 402, 741

Harrison, R. A., Sawyer, E. C., Carter, M. K., et al. 1995, Sol. Phys., 162,233

Harrison, R. A., Lang, J., Brooks, D. H., \& Innes, D. E. 1999, A\&A, 351,1115

Harrison, R. A., Hood, A. W., \& Pike, C. D. 2002, A\&A, 392, 319

Innes, D. E., \& Tóth, G. 1999, Sol. Phys., 185, 127

Innes, D. E., Inhester, B., Axford, W. I., \& Wilhelm, K. 1997, Nature, 386,811
Kjeldseth Moe, O., \& Nicolas, K. R. 1977, ApJ, 211, 579

Marik, D., \& Erdélyi, R. 2002, A\&A, 393, L73

McClymont, A. N. 1989, ApJ, 347, L47

Pérez, M. E., Doyle, J. G., Erdélyi, R., \& Sarro, L. M. 1999, A\&A, 342,279

Peter, H. 2000, A\&A, 360, 761

Peter, H. 2001, A\&A, 374, 1108

Peter, H., \& Judge, P. G. 1999, ApJ, 522, 1148

Priest, E. R., Hood, A. W., \& Bewsher, D. 2002, Sol. Phys., 205, 249

Roussev, I., \& Galsgaard, K. 2002, A\&A, 383, 697

Roussev, I., Galsgaard, K., Erdélyi, R., \& Doyle, J. G. 2001a, A\&A, 370, 298

Roussev, I., Doyle, J. G., Galsgaard, K., \& Erdélyi, R. 2001b, A\&A, 380, 719

Ryutova, M. P., \& Tarbell, T. D. 2000, ApJ, 541, L29

Sarro, L. M., Erdélyi, R., Doyle, J. G., \& Pérez, M. E. 1999, A\&A, 351,721

Spadaro, D., Lanza, A. F., Lanzafame, A. C., et al. 2003, ApJ, 582, 486

Stix, M. 2002, The Sun, 2nd edition (Springer-Verlag)

Teriaca, L., Doyle, J. G., Erdélyi, R., \& Sarro, L. M. 1999, A\&A, 352, L99

Wilhelm, K., Curdt, W., Marsch, E., et al. 1995, Sol. Phys., 162, 189

Winebarger, A. M., Updike, A. C., \& Reeves, K. K. 2002, ApJ, 570, L105 